Delta-sigma modulation microphone sensors employing a resonant tunneling diode with a suspended microstrip resonator

\begin{tabular}{|r|l|}
\hline Journal: & Sensor Review \\
\hline Manuscript ID & SR-03-2020-0044.R1 \\
\hline Manuscript Type: & Original Manuscript \\
\hline Keywords: & $\begin{array}{l}\text { delta-sigma modulator, resonant tunneling diode, ultrasonic sensor, } \\
\text { suspended microstrip resonator }\end{array}$ \\
\hline
\end{tabular}

SCHOLARONE ${ }^{m}$

Manuscripts 


\title{
Delta-sigma modulation microphone sensors employing a resonant tunneling diode with a suspended microstrip resonator
}

\begin{abstract}
Purpose of this paper - We propose and demonstrate novel microphone sensors based on the frequency delta-sigma modulation (FDSM) technique, which replaces the conventional delta-sigma modulator in the delta-sigma analog-todigital converters. A key of the FDSM technology is to employ a voltagecontrolled oscillator ( $\mathrm{VCO}$ ) for converting an input analog signal to a 1-bit pulse-density modulated digital signal. High-performance sensors can be realized if the VCO is replaced by an oscillator whose oscillation frequency depends on an external physical parameter.

Design/methodology/approach - Microphone sensors are proposed based on FDSM that employs a suspended microstrip disk resonator, where the backside ground plane is replaced by a thin metal diaphragm. A resonant tunneling diode (RTD) oscillator is also employed, as the performance of these sensors significantly depends on the oscillation frequency. To demonstrate the basic operation of the proposal, prototype devices were fabricated with an InGaAs/AlAs RTD.

Findings - A satisfactory noise shaping property, which is a significant nature of delta-sigma modulation, was demonstrated over three decades for the prototype device. A sound-sensing peak was also clearly observed when applying 1 $\mathrm{kHz}$ sound from a speaker.

Practical implications - High-performance ultrasonic microphone sensors can be realized if we fabricate the sensors using a thin InP substrate with highfrequency oscillator design.

What is original/value of paper - In this study, we proposed and experimentally demonstrated novel microphone sensors, which are promising as future ultrasonic sensors that have high dynamic range with wide bandwidth.
\end{abstract}

\section{Keywords}

Delta-sigma modulator, resonant tunneling diode, suspended microstrip resonator, ultrasonic sensor 


\section{Introduction}

Delta-sigma modulation, which is a technique for performing analog-to-digital conversion (ADC), offers a wide dynamic range and high signal-to-noise ratio (SNR) without using high-precision analog components (Candy, 1974; Norsworthy et al., 1996; Pavan et al., 2017). A conventional delta-sigma modulator (DSM) comprises an integrator, 1-bit quantizer, and feedback digital-to-analog converter (DAC). Among them, the integrator and feedback DAC limit the sampling rate and, thus, the bandwidth. Recently, frequency DSMs (FDSMs) based on a voltage-controlled oscillator (VCO) have been attracting considerable attention (Høvin et al., 1997; Iwata et al., 1999; Maezawa et al., 2008; Straayer et al., 2008; Li et al., 2019) because they require no integrator or feedback DAC and can be operated at significantly high frequencies.

FDSMs can also be used for realizing high-performance digital output sensors when the VCO is replaced by an oscillator whose oscillation frequency depends on an external physical parameter. A strain sensor and digital microphone were recently proposed by employing FDSM (Tajika et al., 2016; Fujino et al., 2013; Maezawa et al., 2016a). Because the performance of FDSM sensors significantly depends on the oscillation frequency, using compound semiconductor high-frequency devices in the oscillator offers considerable advantages. In this study, we propose FDSM microphone sensors based on a resonant-tunneling diode (RTD) oscillator. An RTD is an ultrahigh-frequency device, and an oscillation frequency close to $2 \mathrm{THz}$ has been demonstrated for RTD oscillators (Maekawa et al., 2016; Asada et al., 2016; Izumi et al., 2017). FDSM sensors that use RTD oscillators might offer a wide frequency bandwidth, high dynamic range, and large SNR owing to their remarkable high-frequency characteristics.

This paper is organized as follows. Section 2 explains the operating principle of FDSM and its application in sensors. FDSM microphone sensors based on an RTD oscillator are also proposed using a suspended microstrip disk resonator. Section 3 describes the design, fabrication, and measured results of the prototype devices fabricated on an FR-4 PCB substrate. Finally, section 4 summarizes this paper.

\section{FDSM and its application in sensors}

\subsection{Frequency delta-sigma modulation}

In this subsection, the delta-sigma analog-to-digital converter (DSADC) and FDSM are briefly explained. Figure 1 (a) shows the basic block diagram of DSADC. It comprises a DSM and decimation filter. The DSM converts the input analog signal to 1-bit pulse-density modulated (PDM) digital signal at a sampling rate $f_{\mathrm{S}}$, which is substantially higher than the Nyquist rate $f_{\mathrm{N}}$. The DSM reduces the low-frequency quantization noise at the expense of its increase in high frequencies. Here, we focus on a first-order DSM, which can be easily implemented using a VCO. In this case, the quantization noise reduces by 20 $\mathrm{dB} /$ dec as the frequency decreases, and this is called first-order noise shaping. The decimation filter removes the high-frequency noise components of the PDM signal and converts it to multi-bit, parallel digital signals at the Nyquist rate. FDSM, which is a simple implementation of DSM, is shown in Fig. 1 (b). It 
(a)

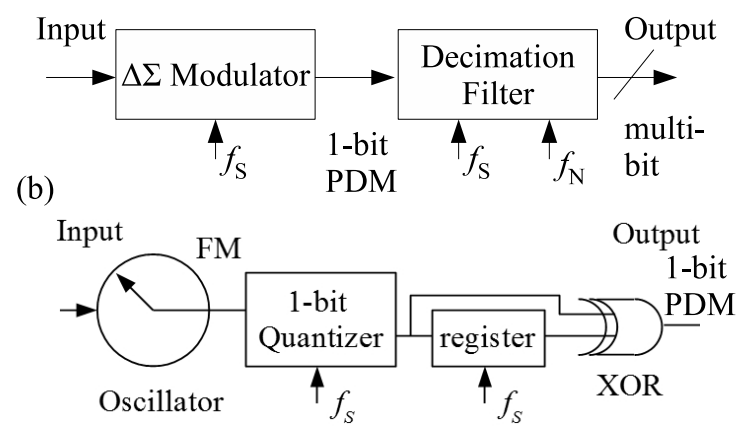

Figure 1: Block diagram of the delta-sigma ADC (a), and the FDSM (b).

comprises a VCO, whose oscillation frequency depends on the input signal, and a small digital circuit that functions as an edge detector. The VCO converts the input analog signal to a frequency-modulated (FM) signal, and the edgedetector circuit outputs pulses when the FM signal crosses zero. Because of the nature of an FM signal, a first-order DSM can be implemented using this simple circuit (Høvin et al., 1997). FDSM offers a significant advantage in that it does not have any feed-back loop, which limits the operating frequency of conventional DSMs.

FDSM is also promising in sensor applications. By replacing the VCO with an oscillator whose oscillation frequency depends on an external physical parameter, it can be used for a sensor integrated with an ADC. The performance of this sensor depends on the frequency modulation width of the oscillator and sampling frequency. Generally, the frequency modulation ratio to the sensing parameter variation is constant, and thus increasing the oscillation frequency is advantageous. Additionally, using a higher-frequency oscillator offers various advantages, including wide frequency bandwidth and small area. Consequently, applying RTD oscillators to these sensors provides significant advantages.

\subsection{Microphone sensors based on a suspended microstrip disk resonator}

We propose microphone sensors that are based on a suspended microstrip disk resonator. Figure 2 depicts the diagram of the suspended microstrip resonator for the proposed sensor, as well as the current distribution on the resonator. The disk-shaped resonator works similar to a $\lambda / 4$ transmission line, as shown in the current distribution. This circuit can be considered a simple LC-parallel, negative-resistance oscillator because the disk resonator functions as an LCparallel circuit near the resonant frequency. The RTD acts as a negative resistor and oscillates at the resonant frequency of the disk resonator. Here, we choose the disk-type resonator, as it has a small characteristic impedance with a large Q-factor owing to its good symmetry. The small characteristic impedance ensures harmonic (sinusoidal) oscillation rather than relaxation oscillation when connected to the RTD (Tajika et al., 2016; Maezawa et al., 2016b). Compared with relaxation oscillation, harmonic oscillation offers a significant advantage of 


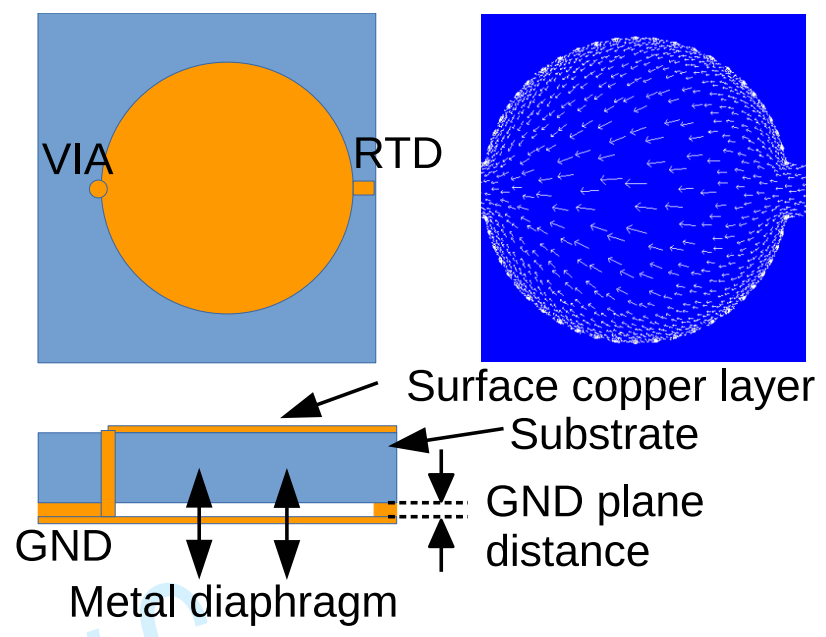

Figure 2: Basic structure of the suspended microstrip resonator based ultrasound sensor using an RTD. (Upper left: top view; lower left: cross-sectional view; right: current distribution of the resonator.

improving the phase noise property. Moreover, a significantly high Q-factor of the disk resonator improves the phase noise, which determines the noise floor of the output data. We inserted a via at the end of the disk to ensure the dc-grounding of the RTD. The key is to replace the backside ground plane with a thin metal diaphragm, which is set apart from the dielectric substrate. The signal-propagation velocity of the (suspended) microstrip line is inversely proportional to the $\sqrt{\epsilon_{\text {eff }}}$, where $\epsilon_{\text {eff }}$ denotes an effective dielectric constant. In the case of the suspended microstrip, the effective dielectric constant is approximated as

$$
\epsilon_{\mathrm{eff}}=\frac{\epsilon_{\mathrm{sub}} \epsilon_{0}}{\epsilon_{0} d_{\mathrm{sub}}+\epsilon_{\mathrm{sub}} d}\left(d_{\mathrm{sub}}+d\right)
$$

where $\epsilon_{\text {sub }}, d_{\text {sub }}$, and $d$ denote the dielectric constant of the substrate, thickness of the substrate, and GND plane distance, respectively. Consequently, the resonant frequency also significantly depends on the GND plane distance.

We performed electromagnetic-field simulation using Keysight EMPro to clarify the dependence of the resonant frequency on the GND plane distance in the case of the suspended microstrip disk based resonators on an InP substrate. An example of the calculated resonant frequency is shown in Fig. 3. The disk diameter was chosen to be $1800 \mu \mathrm{m}$, and the substrate thickness was $450 \mu \mathrm{m}$. The oscillation frequency for the GND plane (diaphragm) distance of $0 \mu \mathrm{m}$, which corresponds to the microstrip resonator, was $9.25 \mathrm{GHz}$. A low resonant frequency was chosen in this example to suppress the effects of the substrate mode. The substrate mode is a resonant mode of the electromagnetic wave in a substrate perpendicular to the surface. For example, the quarter of the wavelength of a $47-\mathrm{GHz}$ electromagnetic wave is equal to the InP-substrate thickness, significantly affecting the S-parameter of the resonator and hindering appropriate oscillation. The resonant frequency increases with the GND plane distance because of decrease in the effective dielectric constant, thereby resulting 


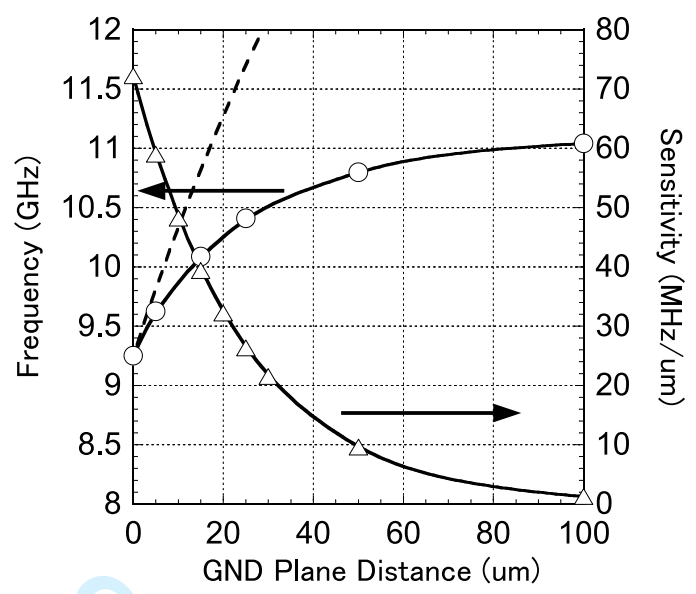

Figure 3: Example of the calculated resonant frequency and sensitivity of the suspended-disk-shaped resonator fabricated on an InP substrate. The resonator diameter is $1800 \mu \mathrm{m}$, and the substrate thickness is $450 \mu \mathrm{m}$. The dashed line shows the resonant frequency for the two-layer dielectric model expressed in equation (1).

in higher signal velocity. The result for the two-layer dielectric model shown in equation (1) is also plotted for reference. As depicted in the figure, this simple model is valid only for small GND plane distances, and it rapidly deviates from the electromagnetic-simulation result. This deviation is attributed to the edge effect, in which the electromagnetic field spreads out of the disk.

This figure also shows the sensitivity of the sensor. The sensitivity is defined as the derivative of the resonant frequency with respect to the GND plane distance. It increases as the GND plane distance decreases. For example, the sensitivities of 50 and $10 \mathrm{MHz} / \mu \mathrm{m}$ were obtained for the distances of 10 and 50 $\mu \mathrm{m}$, respectively. These values are significantly higher than that of a cylindrical cavity resonator sensor, i.e., $0.27 \mathrm{MHz} / \mu \mathrm{m}$ (Maezawa et al., 2016a). Such high sensitivities are essential for high-performance sensors. In addition, this sensor structure is advantageous for integration process.

Upon reducing the substrate thickness, a resonator with higher frequency can be used without interference with the substrate mode. Figure 4 shows such an example. It depicts the simulation results for a resonator fabricated on a $50-\mu \mathrm{m}$-thick InP substrate. The resonator diameter was chosen to be $200 \mu \mathrm{m}$, meaning that the aspect ratio is the same as that for the previous model. The resonant frequency for the microstrip resonator was $94.6 \mathrm{GHz}$, and it increased while moving the GND plane further from the backside of the substrate. Using this thin substrate, a significant variation that exceeded $100 \%$ was observed in the resonant frequency for the GND plane motion of $100 \mu \mathrm{m}$. Because of this substantial variation, the sensitivity became considerably higher than that in Fig. 3. Notably, the sensitivities of 3000 and $400 \mathrm{MHz} / \mu \mathrm{m}$ are expected for the GND plane distances of 10 and $50 \mu \mathrm{m}$, respectively. Additionally, such a significant variation in the oscillation frequency is possible for RTD oscillators because RTDs show a nearly constant negative differential conductance for up 


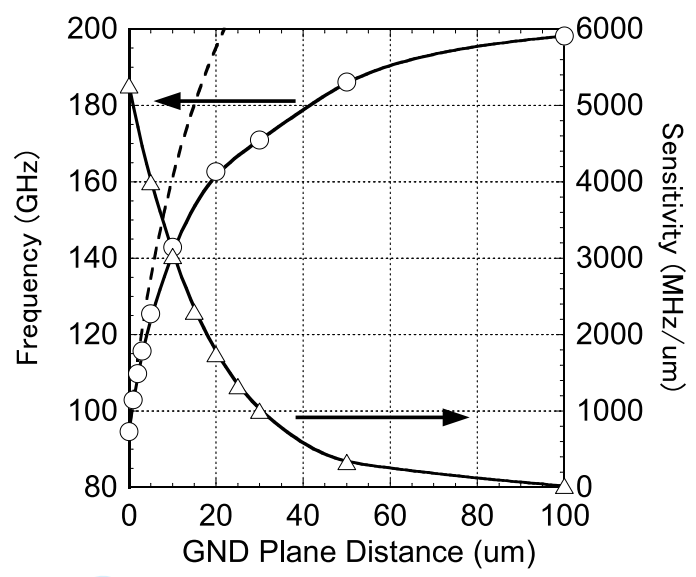

Figure 4: Example of the sensitivity calculated for the suspended-disk-shaped resonator fabricated on an $\mathrm{InP}$ substrate. The resonator diameter is $200 \mu \mathrm{m}$, and the substrate thickness is $50 \mu \mathrm{m}$. The dashed line represents the resonant frequency for the two-layer dielectric model described in equation (1).

to the $\mathrm{THz}$ frequency range.

Here, we compare our proposal with MEMS capacitive microphones (Shah et al., 2019; Zawawi et al., 2020), which are widely used because of their high sensitivity, flat frequency response, and low noise levels. The sensitivity of capacitive microphones is defined as the output voltage swing for the sound pressure of $1 \mathrm{~Pa}$, and it ranges between a few $\mathrm{mV} / \mathrm{Pa}$ and few tens of $\mathrm{mV} / \mathrm{Pa}$. Another important figure of merit, i.e., SNR, is defined for the sound pressure of $1 \mathrm{~Pa}$ and ranges between 60 and $70 \mathrm{~dB}$ for the bandwidth of $20 \mathrm{kHz}$. The definition of the sensitivity of our device is fairly different than that of an MEMS capacitive microphone, and thus it is difficult to directly compare them. Instead, we compare them in terms of SNR. Notably, the SNR of FDSM can be expressed as (Høvin et al., 1997) follows:

$$
\mathrm{SNR}=20 \log \left(\frac{\Delta f}{f_{\mathrm{s}} \sqrt{2}}\right)-20 \log \left(\frac{\pi}{6}\left(\frac{2 f_{\max }}{f_{\mathrm{s}}}\right)^{3 / 2}\right),
$$

where $\Delta f, f_{\mathrm{s}}$, and $f_{\max }$ denote the maximum deviation in the frequency, sampling rate, and bandwidth, respectively. Assuming that the diaphragm-motion amplitude is $0.5 \mu \mathrm{m}$ for the sound pressure of $1 \mathrm{~Pa}$, as that for a typical MEMS microphone, we obtain a high SNR of $107 \mathrm{~dB}$ for the device shown in Fig. 3 while assuming a sampling rate of $12.6 \mathrm{GHz}$. The SNR can be further increased to $153 \mathrm{~dB}$ for the device shown in Fig. 4 while assuming a sampling rate of 126 GHz. These high SNRs indicate the promise shown by FDSM microphones. Moreover, FDSM microphones offer an important advantage in that they do not suffer from pull-in instability. This instability occurs in MEMS capacitive microphones when the applied voltage exceeds a critical value, and thus the electric field snaps the diaphragm down to the back plate. This restricts the maximum voltage, minimum air-gap, and, hence, sensitivity. Because an FDSM microphone requires no DC bias, it does not suffer from pull-in instability. 
Finally, we comment on the amplitude of the diaphragm motion under a sound pressure. Upon reducing the device size to increase the frequency, the diaphragm-motion amplitude also decreases. However, the diaphragm size is independent of disk size, and one can shrink the disk while maintaining the diaphragm diameter. Moreover, the GND plane distance can be reduced without the risk of pull-in instability to compensate for a small diaphragm motion.

Consequently, the oscillators that use suspended microstrip resonators with an RTD might show significantly high sensitivity and are thus promising for use in microphone sensors. Especially, such high-frequency oscillators are promising for use in ultrasonic sensors.

\section{Demonstration of the basic operation using prototype devices}

Fabricating the sensor using an InP substrate requires precise and cumbersome processes, including substrate thinning, via-hole construction, and diaphragm formation. Here, we fabricated prototype devices using an FR-4 PCB substrate with InGaAs/AlAs RTD chip. Although these devices have lower sensitivity than that of the above-mentioned sensors because of their lower oscillation frequency and lower dielectric constant, they can perform the basic operation and provide us with fundamental data to discuss their performances.

\subsection{Prototype device structure and fabrication process}

Figure 5 depicts the structure and circuit diagram of the prototype device we fabricated. We used a 0.8-mm-thick FR-4 PCB substrate, whose dielectric constant was approximately 4.5 at $1 \mathrm{GHz}$. The thickness of the surface copper layer was $18 \mu \mathrm{m}$. The RTDs were prepared as follows. First, the epitaxial layers were grown via molecular-beam epitaxy on a GaAs substrate with metamorphic buffer layer (Maezawa et al., 2005). The RTDs were then fabricated via conventional photolithography and the lift-off process. Subsequently, a $2.5 \times 2.5 \mathrm{~mm}^{2}$ RTD chip was cut out from the substrate and bonded to the FR- 4 substrate. $\mathrm{Al}$ wires with the diameter of $25 \mu \mathrm{m}$ were used to connect the RTD pads to the copper lines on the PCB. The parameters of the fabricated RTD are listed in Table 1 . The metal diaphragm was made of a commercial, 12- $\mu$ m-thick Al-foil. The disk diameter was $20 \mathrm{~mm}$, which corresponds to the resonant frequency of $1.25 \mathrm{GHz}$ when the backside metal completely contacts the substrate (microstrip case). This low frequency of $1.25 \mathrm{GHz}$ was chosen to eliminate the influence of bonding wires. The FR-4 substrate was processed using a PCB milling machine so that the backside of the disk resonator could be slightly thinned while removing the copper. The ventilators were fabricated around the resonator to eliminate the effects due to variation in the ambient air pressure. The oscillator comprised a disk resonator and an RTD connected in series. The bias terminal of the RTD was grounded using a $5-\Omega$ resistor to suppress spurious oscillations. An image of the fabricated device is shown in Fig. 6. 

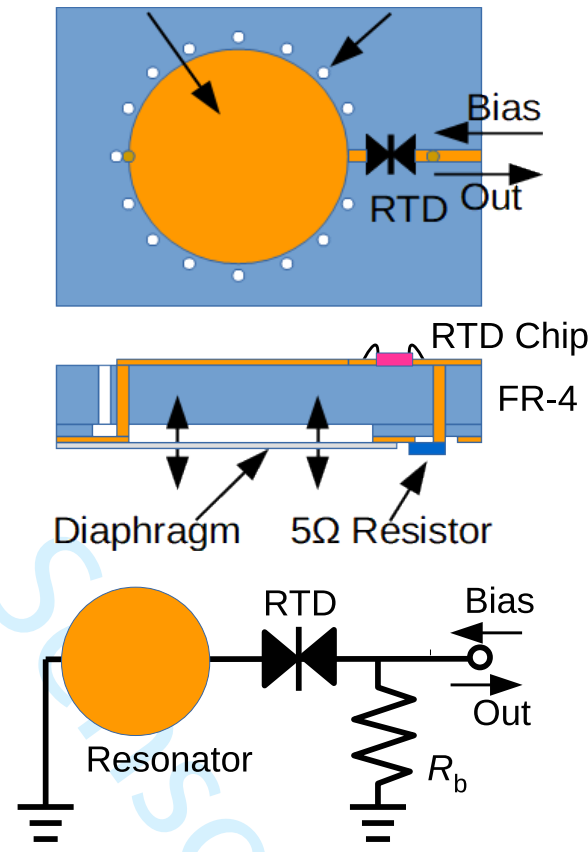

Figure 5: Structure and circuit diagram of the sensor fabricated using an RTD.

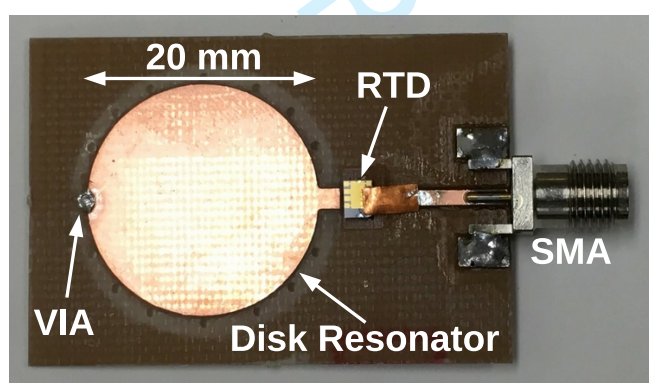

Figure 6: Fabricated sensor. The stabilizing resistor, $R_{\mathrm{b}}$, is on the backside of the substrate.

Table 1: Parameters of the RTD used in the experiment.

\begin{tabular}{c|c}
\hline Peak voltage & $0.67 \mathrm{~V}$ \\
\hline Peak current density & $1.0 \times 10^{5} \mathrm{~A} / \mathrm{cm}^{2}$ \\
\hline Area & $18 \mu \mathrm{m}^{2}$ \\
\hline Peak/valley ratio & 6 \\
\hline
\end{tabular}

\subsection{Microstrip resonator oscillator}

First, we tested an oscillator without a diaphragm, but with a backside GND plane that contacted the substrate, for reference. The oscillating frequency of 


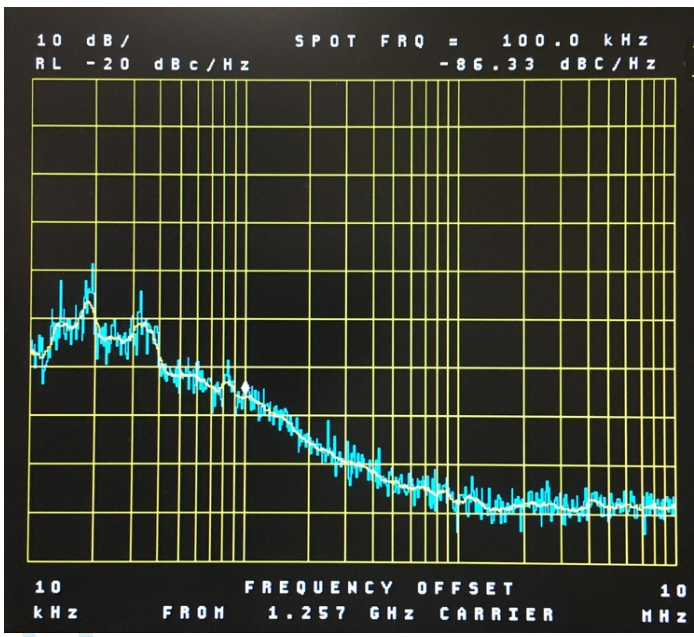

Figure 7: Phase noise property of the fabricated microstrip disk resonator oscillator.

the oscillator was approximately $1.25 \mathrm{GHz}$, which was stable against changes in the bias voltage (less than $0.05 \mathrm{GHz}$ over the bias-voltage range of $0.7-1.2 \mathrm{~V}$ ). This implies that the harmonic-type oscillation occurred because the relaxationtype oscillation significantly depends on the bias voltage (Tajika et al., 2016). Next, we measured the phase noise property of the oscillator, as shown in Fig. 7. A low phase noise of $-86 \mathrm{dBc} / \mathrm{Hz}$ was obtained at $100 \mathrm{kHz}$ apart from the carrier frequency.

\subsection{Experimental setup for sound sensing}

Figure 8 shows the setup of the sound-sensing experiment. The bias voltage was applied via a bias-tee, and the oscillation signal was also taken out from the bias-tee. The signal was amplified using an RF amplifier (Keysight 83017A) and fed to a signal analyzer that was constructed on a field-programmable gate array (FPGA) chip. A 2-GHz, low-pass filter was inserted between the amplifier and signal analyzer. It was essential because the output of the oscillator was taken from the bias port. Accordingly, the output voltage was proportional to the RTD current, which has strong harmonics because of the strong non-linear $I-V$ characteristics of RTD, although the oscillation in the resonator is sinusoidal.

The signal-analyzer circuit was constructed on an FPGA evaluation board, called Xilinx ZCU-102 (XC7K325T-2FFG900C). Its block diagram is shown in Figure 9. It comprises a high-frequency sampler, an edge detector, and a digitalfilter. A high-frequency transceiver module was used for the sampling circuit, which could be operated at the sampling rate of as high as $16.3 \mathrm{~Gb} / \mathrm{s}$. Here, the sampling rate was chosen to be $12.582912 \mathrm{~Gb} / \mathrm{s}$, which corresponds to the oversampling ratio of 65536 for a signal bandwidth of $96 \mathrm{kHz}$.

The sampled 1-bit digital signal was converted to a 32-bit parallel data stream and then fed to the edge detector, which comprised XORs and registers. Subsequently, the 32-bit PDM data stream was compressed to a 6-bit data 


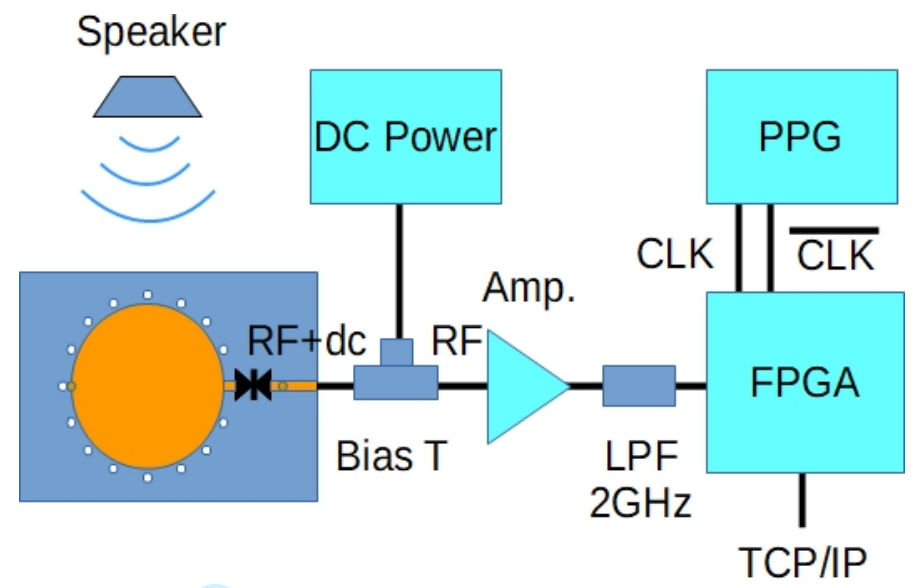

Figure 8: Measurement setup of the sound-sensing experiments.

stream by counting " 1 ". This works as a moving average LPF. Next, the 6bit data stream was fed to the cascaded integrator-comb (CIC) filter, which functioned as a sinc2 filter, to eliminate high-frequency noise components and convert the signal to a 32-bit output data stream at the Nyquist rate. The filter module is shown in the lower illustration in Fig. 9. The output data were transferred to the random access memory by using a direct memory access (DMA) module. Finally, the output signal was displayed on a PC. When we tested the noise shaping property, the output of the edge detector was directly transferred to the memory.

\subsection{Results and discussion}

To check the natural gap between the substrate and diaphragm, we first attached a fixed and flat GND plane (copper-covered PCB) to the backside of the substrate, following which we measured the oscillation frequency. The circuit oscillated at approximately $1.63 \mathrm{GHz}$. We compared this oscillation frequency with that of the electromagnetic-simulation result and obtained a gap of approximately $0.15 \mathrm{~mm}$, which was significantly larger than the copper-layer thickness of $18 \mu \mathrm{m}$. This gap corresponded to the scraped depth of the substrate when the backside copper was removed using the PCB milling machine.

Next, the fixed GND plane was replaced by a $12-\mu$ m-thick Al-foil. The oscillation frequency was approximately $1.54 \mathrm{GHz}$, which was slightly smaller than that of the fixed GND plane. This difference is probably because of the distortion of the Al-foil.

Subsequently, we measured the response of the fabricated sensor to an audio sound signal generated from a speaker. Figure. 10 shows the noise shaping property. This is a fast Fourier transformation (FFT) result of the 1-bit PDM digital signal, which was directly taken from the edge detector. The Blackman-Harris window was used for the FFT. A clear noise-shaping behavior was observed over three decades, thereby demonstrating an appropriate delta-sigma operation. However, the slope gradually decreased for frequencies less than $10 \mathrm{MHz}$, and a noise floor was observed at the frequencies lower than $1 \mathrm{MHz}$. This noise 


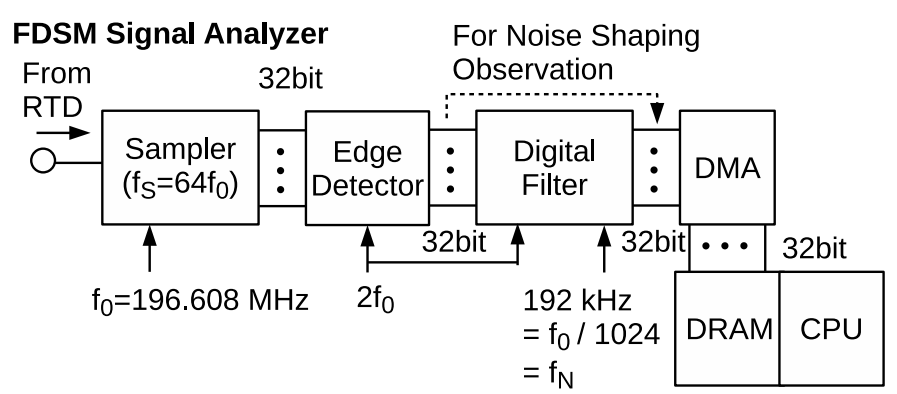

Digital Filter

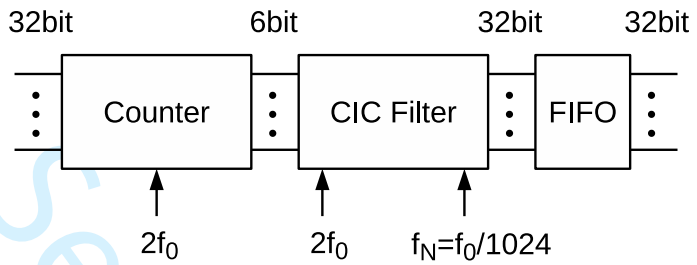

Figure 9: Block diagram of the signal-analyzer circuit on an FPGA. The lower illustration details the digital filter, which eliminates the high-frequency noise components and converts the PDM signal to a 32-bit parallel signal at the Nyquist rate.

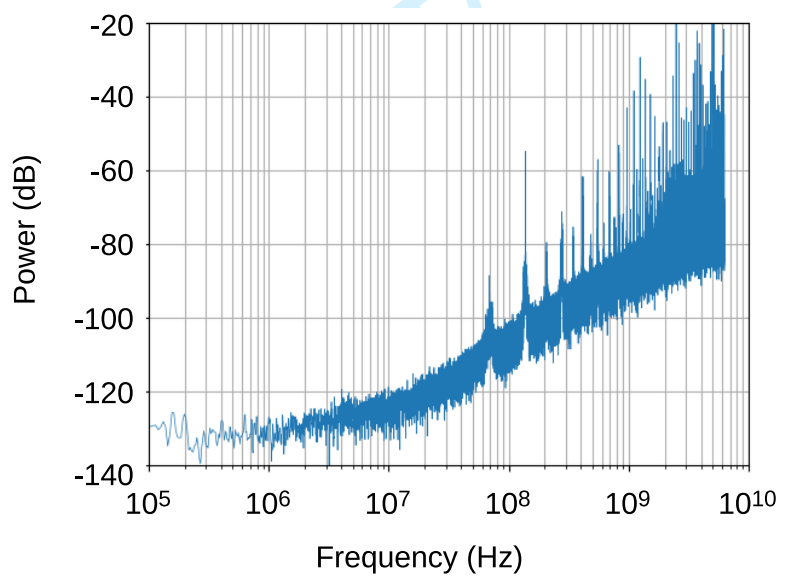

Figure 10: Example of the FFT result of the output 1-bit digital signal, which was obtained immediately after edge detection. Four data series were averaged for this plot.

floor is attributed to the phase noise of the oscillator resulted form the significant dielectric loss of the FR-4 substrate. This should be improved using an InP substrate. Furthermore, many peaks are visible at frequencies higher than $100 \mathrm{MHz}$. They are probably attributed to the pattern noise.

An example of the FFT results for the output 32-bit data stream at the 


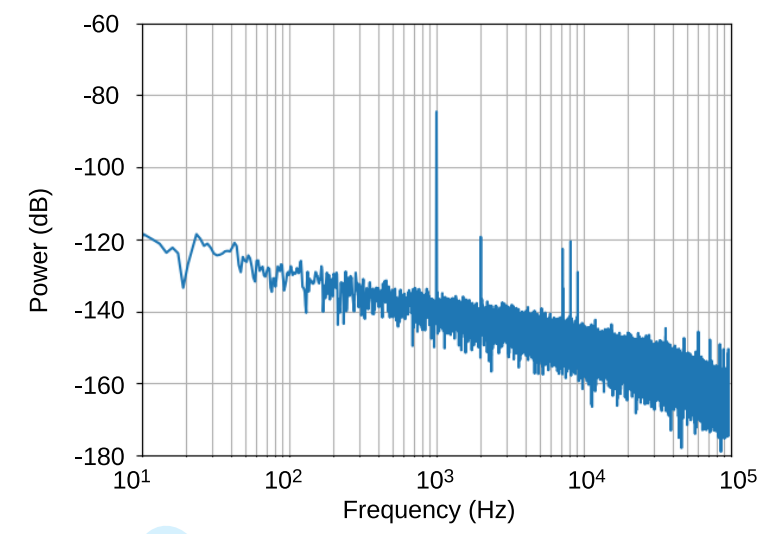

Figure 11: Example of the FFT results of the digital output stream from the digital filter. A 1-kHz sound signal with $114 \mathrm{~dB}$ SPL was applied to the sensor. The frequency band width was $96 \mathrm{kHz}$, and hence the Nyquist rate was 192 $\mathrm{kHz}$.

Nyquist rate $(192 \mathrm{kHz})$ is shown in Fig. 11. Here, we used a special type of speaker, a calibrator for sound-level meter. It could apply a $1-\mathrm{kHz}$ sound with an accurate pressure of $114 \mathrm{~dB}$ SPL when hermetically connected to the microphone. A clear sound-signal peak was observed at $1 \mathrm{kHz}$; the peak-topeak frequency modulation amplitude was approximately $500 \mathrm{kHz}$ for this peak. The electromagnetic simulation of this device revealed that its sensitivity was $0.94 \mathrm{MHz} / \mu \mathrm{m}$, which indicated that the average diaphragm-vibration amplitude was approximately $0.5 \mu \mathrm{m}$. FDSM sensors can handle this small motion of the diaphragm with a wide bandwidth. Notably, the small vibration amplitude of this device is attributed to the thick $\mathrm{Al}$ diaphragm, and it can be enhanced further using a thinner diaphragm.

It is noted here that one must consider the skin depth when reducing the diaphragm thickness. The microwave electromagnetic field penetrates a metal at skin depth. The diaphragm thickness should be a few times greater than the skin depth to confine the electromagnetic waves. Because the skin depth of the Al-film is $2.2 \mu \mathrm{m}$ for $1.5 \mathrm{GHz}$, we can reduce the thickness by half. Moreover, we can further reduce the thickness to less than $1 \mu \mathrm{m}$ upon using higher frequencies, as the skin depth is inversely proportional to the square root of frequency. Thus, we can improve the sensitivity and enable the detection of a significantly low pressure.

The sensitivity to the unit motion of the diaphragm is also significantly lower than the values discussed in Section 2. This is attributed to the low dielectric constant of the PCB substrate, large gap, and low oscillation frequency. A considerable peak height, for example, 60-dB higher peak, can be expected upon using the 50- $\mu$ m-thick InP substrate. Moreover, using the InP substrate offers another advantage, i.e., of reduced noise floor. The Q-factor of the resonator significantly depends on the dielectric loss tangent $(\tan \delta)$. The $\tan \delta$ of $\mathrm{InP}$ is approximately 0.001 , while that of FR-4 is 0.01 . Therefore, we ex- 
pect a reduction of $20 \mathrm{~dB}$ in the phase noise and that of $20 \mathrm{~dB}$ in the noise floor. Consequently, significantly high performance is expected using the InP substrate.

Throughout the experiment, the dc-bias voltage was set to $1.0 \mathrm{~V}$, which corresponds to the power consumption of approximately $10 \mathrm{~mW}$ in the RTD. However, the bias-stabilization resistor $R_{\mathrm{b}}$ unnecessarily consumes a significantly higher power of $200 \mathrm{~mW}$, which can be eliminated using hard-type oscillator technique (Maezawa et al., 2018; Narahara and Maezawa, 2005).

Finally, we comment on the non-linearity of this sensor. FDSM-type ADCs suffer from VCO non-linearity. In this application, we observed no noticeable degradation due to the non-linearity. (The 2nd harmonic peak in Fig. 11 should be attributed to the speaker because other microphones also show this peak.) This is because the frequency modulation amplitude is only approximately $0.03 \%$ of the oscillation frequency. However, this small ratio renders the sensitivity low. This can be easily increased by increasing the oscillation frequency while maintaining the modulation ratio as low. This is one of the most important advantages of using high-frequency oscillators.

\section{Conclusion}

FDSM microphone sensors were proposed using an RTD oscillator. First, we discussed the advantages of the FDSM technique for realizing high-performance sensors, as well as the advantages of using RTD oscillators in FDSM sensors. The microphone sensors that used a suspended microstrip disk resonator were proposed based on the above discussion. The backside ground plane of the suspended microstrip resonator was replaced by a thin metal diaphragm, which modulated the oscillation frequency of the RTD oscillator. Through electromagnetic-field simulation, we demonstrated that high sensitivity of the oscillation frequency to the diaphragm motion could be obtained with these structures.

A prototype device was fabricated on an FR-4 PCB substrate using an InGaAs/AlAs RTD. To demonstrate the FDSM operation, we constructed an FPGA-based signal-analyzer circuit, which could sample the output of the RTD oscillator with the sampling rate as high as $16.3 \mathrm{GS} / \mathrm{s}$. Using this signal analyzer, a satisfactory noise shaping property over three decades and sound sensing were demonstrated for the prototype device. In conclusion, high-performance ultrasound microphone sensors should be realized using thin InP substrates with high-frequency oscillators.

\section{References}

Asada, M., and Suzuki, S., (2016), "Room-Temperature Oscillation of Resonant Tunneling Diodes close to $2 \mathrm{THz}$ and Their Functions for Various Applications," J. Infrared Milli Terahertz Waves, DOI $10.1007 / \mathrm{s} 10762-016-0321-6$.

Candy, C., (1974), "A Use of Limit Cycle Oscillations to Obtain Robust Analog-to-Digital Converters," IEEE Trans. on Commun., vol. 22, no. 3, pp. 298-305.

Fujino, S., Mizuno, Y., Takaoka, K., Nakano, J., Mori, M., and Maezawa, K., (2013), "Experimental Demonstration of Noise Shaping in the Digital Microphone Employing Frequency $\Delta \Sigma$ Modulation," IEICE Trans. Electron. (Japanese Edition), Vol. J96-C, No. 12, pp. 554-555. 
Høvin, M., Lande, T. S., Toumazou, C. (1997), "Delta-Sigma Modulators Using FrequencyModulated Intermediate Values," IEEE J. Solid-State Circuits 32, pp. 13-22.

Iwata, A., Sakimura, N., Nagata, M., and Morie, T., (1999), "The Architecture of Delta Sigma Analog-to-Digital Converters Using a Voltage-Controlled Oscillator as a Multibit Quantizer," IEEE Trans. Circuits and Systems-II 46, pp. 941-945.

Izumi, R., Suzuki, S., and Asada, M., (2017), "1.98 THz Resonant-Tunneling-Diode Oscillator With Reduced Conduction Loss By Thick Antenna Electrode," Int. Conf. Infrared, Millimeter, and Terahertz waves (IRMMW-THz), MA3.1, Cancun, Mexico.

Li, S., Sanyal, A., Lee, K., Yoon, Y., Tang, X., Zhong, Y., Ragab, K., and Sun, N., (2019), "Advances in Voltage-Controlled-Oscillator-Based $\Delta \Sigma$ ADCs," IEICE TRANS. ELECTRON., VOL.E102-C, NO.7, pp. 509-519.

Maekawa, T., Kanaya, H., Suzuki, S., and Asada, M., (2016), "Oscillations up to 1.92 THz in resonant tunneling diode by reduced conduction loss," Appl. Phys. Exp., vol. 9, 024101.

Maezawa, K., Fujino, S., Yamaoka, T., and Mori, M., (2016a), "Delta Sigma Modulation Microphone Sensors Using a Microwave Cavity Resonator," Electron. Lett. 52, pp. 1651-1652. DOI $10.1049 /$ el.2016.2538

Maezawa, K., Iwase, T., Ohno, Y., Kishimoto, S., Mizutani, T., Sano, K., Takakusaki, M., and Nakata, H., (2005), "Metamorphic Resonant Tunneling Diodes and Its Application to Chaos Generator ICs," Jpn. J. Appl. Phys., Vol. 44, No. 7A, pp. 4790-4794.

Maezawa, K., and Mori, M., (2016b), "Impulse Sensitivity Function Study of the Phase Noise in Resonant Tunneling Diode Oscillators," Asia-Pacific Workshop on Fund. and Appl. Semicond. Dev. (AWAD2016), Hakodate, Japan.

Maezawa, K., and Mori, M., (2018), "Possibilities of Large Voltage Swing Hard-Type Oscillators Based on Series-Connected Resonant Tunneling Diodes," IEICE Trans. Electon., Vol.E101-C, No. 5, pp. 305-310.

Maezawa, K., Sakou, M., Matsubara, W., and Mizutani, T., (2006), "Resonant tunneling deltasigma modulator suitable for high-speed operation," Electron. Lett. 42, 20063215.

Narahara. K., and Maezawa, K., (2018), "Characterization of a hard-type oscillator using series-connected tunnel diodes," IEICE Electron. Express, Vol. 15, No. 10, pp. 1-6. DOI $10.1587 /$ elex.15.20180355

Norsworthy, S. R., Schreier, R., Temes, G. C. (1996), Delta-Sigma Data Converters, IEEE Press, New York, USA.

Shah, M. A., Shah, I. A., Lee, D.-G., and Hur, S., "Design Approaches of MEMS Microphones for Enhanced Performance", Journal of Sensors, Vol. 2019, 9294528, DOI: 10.1155/2019/9294528

Pavan, S., R., Schreier, R., Temes, G. C. (2017), "Understanding Delta-Sigma Data Converters (2nd Ed.)," Willey \& Sons, New Jersey, USA.

Straayer, M. Z., and Perrott, M. H., (2008), "A 12-bit, 10-MHz bandwidth, continuous- time ADC with a 5-bit, 950-MS/s VCO-based quantizer," IEEE J. Solid-State Circ. 43, p. 805.

Tajika, T., Kakutani, Y., Mori, M., and Maezawa, K., (2016), "Experimental demonstration of strain detection using resonant tunneling delta-sigma modulation sensors," Phys. Status Solidi, A 214, No. 3, 1600548. DOI: 10.1002/pssa.201600548

Zawawi, S. A, Hamzah, A. A., Majlis, B. Y., and Mohd-Yasin, F., (2020), "A Review of MEMS Capacitive Microphones," Micromachines, 2020, 11, 484, doi:10.3390/mi11050484 